\title{
The time-consuming demands of the practice of medical genetics in the era of advanced genomic testing
}

\author{
Rivka Sukenik-Halevy, MD ${ }^{1,2}$, Mark David Ludman, MD ${ }^{1,3}$, Shay Ben-Shachar, MD ${ }^{2,4}$ and \\ Annick Raas-Rothschild, MD ${ }^{2,5}$
}

\begin{abstract}
Purpose: Clinical genetics services are time- and labor-intensive. With increasing pressure for cost-effective medical care, the means of providing medical genetics services need to be evaluated in the current era of new genomic technologies.
\end{abstract}

Methods: An anonymous online survey regarding activities linked to medical genetics practice was administered to an international cohort of professionals.

Results: Among 151 responses, the reported average time required for pediatric, oncogenetic, pregnancy with a malformed fetus, and preamniocentesis counseling sessions was $48,37,40$, and $18 \mathrm{~min}$, respectively. The time required to prepare a summary letter followed a similar pattern. Professionals with less experience needed more time for specific activities. The time required for the total workup of a pediatric patient ranged from $1 \mathrm{~h}$ and $48 \mathrm{~min}$ to $4 \mathrm{~h}$, most of which was associated with indirect activities. Professionals performing one type of consultation ( $74 \%$ pediatric geneticists) perform fewer consultations per week. Respondents' narrative comments reflected the complexity of the work and challenges faced.

Conclusion: Clinical genetics is a time-consuming profession with increased demands related to advanced genetic and genomic testing. Further consideration is required to determine how to adapt these changes to the demands of cost-effectiveness without compromising the quality of patient care.

Genet Med advance online publication 16 July 2015

Key Words: clinical genetics practice; cost effectiveness; international online survey; new genomic technologies

\section{INTRODUCTION}

Genetics is a rapidly evolving field, with profound advances in technology and bioinformatics. Clinical geneticists and genetic counselors are faced with new challenges and are required to meet the growing demand for genetics services and to adjust to the complex, evolving nature of the practice of medical genetics in this era. Geneticists are often compared with professionals from other medical disciplines in terms of "productivity," based on volume metrics, such as the number of patients seen or the number of patient encounters. The practice requirements of Clinical Genetics, however, which have always differed in terms of the amount of time required to assess patients and provide adequate genetic consultation, have become even more complex and time consuming as a result of new technologies, such as chromosomal microarray analysis (CMA) and next-generation sequencing (NGS), including whole-exome sequencing and gene sequencing panels. In addition to the "old fashioned" clinical examination of patients with rare diseases, interpretation of the clini$\mathrm{cal}$ relevance of the complex data resulting from these tests is a challenge for both laboratory and clinical geneticists. As new genetic technologies such as CMA and NGS become the standard of care in Clinical Genetics, the nature of genetic services provided is changing dramatically. The impact of these tests on the workload of clinical geneticists in terms of their use and interpretation, as well as the task of explaining these tests to families, needs to be assessed.

The purpose of this preliminary study was to evaluate the time requirements resulting from the use of these new technologies and the impact of these changes on the practice of medical geneticists.

\section{MATERIAL AND METHODS}

\section{Data collection}

An online survey was designed to obtain preliminary information regarding the impact of advanced genetic testing on the time and workload of medical geneticists and genetic counselors around the world. The survey was sent to medical geneticists and genetic counselors through the mailing lists of the annual David W. Smith Workshop on Malformations and Morphogenesis, the International Manchester Dysmorphology Conference, and the Israeli Society of Medical Geneticists.

The survey consisted of 10 questions, 9 of which were multiple choice and 1 that provided an option for narrative comments. Professionals were asked to estimate the time required to perform different activities. The choices for the time frames were divided into four groups, in which 1 represented the shortest time range and 4 the longest. The questionnaire is presented in the Supplementary Data online and is available at http://www.surveymonkey.com/s/F2KJV2K.

\footnotetext{
${ }^{1}$ Institute of Genetics, Meir Medical Center, Kfar-Saba, Israel; ${ }^{2}$ Sackler Faculty of Medicine, Tel-Aviv University, Tel Aviv, Israel; ${ }^{3}$ Department of Pediatrics and Medicine, Faculty of Medicine, Dalhousie University, Halifax, Nova Scotia, Canada; ${ }^{4}$ The Genetic Institute, Tel Aviv Sourasky Medical Center, Tel Aviv, Israel; ${ }^{5}$ The Institute for Rare Diseases and Institute of Genetics, Sheba Medical Center, Tel Hashomer, Israel. Correspondence: Rivka Sukenik-Halevy (Riki.sukenik@gmail.com)

Submitted 14 March 2015; accepted 2 June 2015; advance online publication 16 July 2015. doi:10.1038/gim.2015.96
} 


\section{Statistical analysis}

The mean, SD, median, mode, and minimum and maximum values were calculated for each question. A two-tailed sample $t$-test or Mann-Whitney nonparametric test, as appropriate was used to test the differences between any two study groups. One-way analysis of variance was used in the comparison between three categories. $P<0.05$ was considered statistically significant. All statistical analyses were done with SPSS-21 software (SPSS, Inc, Chicago, IL).

\section{RESULTS}

Participants and sample characteristics

Responses were received from 151 professionals: 128 medical geneticists, 2 medical genetics residents, 20 genetic counselors, and 1 clinical psychologist. The number of genetic counselors who responded to our survey was too small for separate analysis.

The majority of the respondents work in Europe $(n=80$; $53 \%)$, and the others are from North America ( $n=43 ; 28.5 \%)$, the Middle East $(n=21 ; 14 \%)$; and Australia and New Zealand $(n=3 ; 2 \%)$. In terms of the seniority of the participants, 25 $(16.7 \%)$ have been practicing genetics for less than 6 years, 30 (20\%) have been in the field for 6-11 years, 51 (34\%) have been practicing for $12-20$ years, and $44(29.3 \%)$ have been in the field for over 20 years.

\section{The time distribution between the different activities} required in the genetic workup for different scenarios

Participants were asked to report the amount of time required for consultations for four different scenarios: a first genetic evaluation session for a pediatric patient, a first counseling session for an oncogenetic patient, a first genetic consultation session for a couple with a malformed fetus detected by ultrasound, and counseling before amniocentesis indicated for maternal

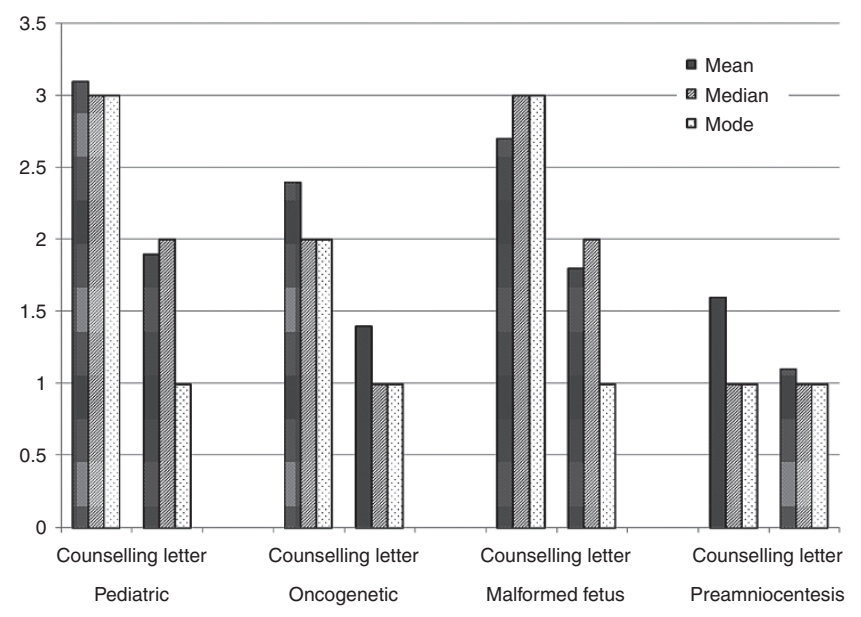

Figure 1 Time required for various counseling situations. The time required for genetic consultation and for summary letters for the following situations: pediatric, oncogenetic, a pregnancy with a malformed fetus, and preamniocentesis counseling sessions. The y axis shows the score according to the options given in the questionnaire: 1 = less than $30 \mathrm{~min}, 2=31-$ $45 \mathrm{~min}, 3=46-60 \mathrm{~min}, 4=$ more than $60 \mathrm{~min}$. age or abnormal biochemical screening. They also were asked to report the time needed to write summary letters for these consultations. Figure 1 depicts the mean, median, and mode for the answers received. Based on a four-point scale, the mean time was longest for pediatric genetic consultation $(3.1 \pm 0.8$, which corresponds to $\sim 48 \mathrm{~min})$, followed by genetic consultation for a fetal malformation $(2.7 \pm 0.8, \sim 40 \mathrm{~min})$, oncogenetic sessions $(2.4 \pm 0.9, \sim 37 \mathrm{~min})$, and counseling before amniocentesis $(1.6 \pm 0.8, \sim 18 \mathrm{~min})$. The median and mode were $46-60 \mathrm{~min}$ for pediatric consultations and consultations for pregnancies with a fetal malformation. These were followed by oncogenetic consultations and counseling before amniocentesis in which the median and mode were 30-45 min and less than $30 \mathrm{~min}$, respectively. Responses regarding counseling letters followed a similar pattern, with the highest mean for pediatric genetics letters and the lowest for preamniocentesis counseling letters. The mode for all consultation letters was less than $30 \mathrm{~min}$.

Participants were asked to evaluate the time needed for certain indirect patient-related activities for the average patient. Table 1 summarizes the responses. Based on the modal value for the answers received, the amount of time required to analyze bioinformatics (NGS and CMA) test results and for literature and database searches related to genetic consultation is $31-60 \mathrm{~min}$ each, according to the survey participants. The reported time required for clinical meetings, consultations regarding issues related to patient care, and attending to bioinformatics issues is between 0 and 30 min each (Table 1).

Based on the mode for the answers received, we calculated the minimum and maximum times required for the total workup of a pediatric patient; this ranged from $1 \mathrm{~h}$ and $48 \mathrm{~min}$ to $4 \mathrm{~h}$. The specifications for this calculation are depicted in Table 2. Regarding pathological CMA results in particular, a mode of 31-45 min was needed to the search the literature and databases, and to provide genetic consultation regarding an affected child or pregnancy.

\section{Time distribution among the different activities}

Most professionals devote the largest portion of their time to clinical work (average mean, median, and mode are $>50 \%$ ). Administrative activities occupy from 11 to $25 \%$ of their time,

Table 1 The amount of time required for indirect activities associated with genetic consultation

\begin{tabular}{|c|c|c|c|}
\hline Activity & Mean $^{a}$ & $\begin{array}{c}\text { Median } \\
(\min )\end{array}$ & $\begin{array}{l}\text { Mode } \\
(\min )\end{array}$ \\
\hline $\begin{array}{l}\text { Literature and database searches related } \\
\text { to counseling }\end{array}$ & $2 \pm 0.7$ & $31-60$ & $31-60$ \\
\hline $\begin{array}{l}\text { Clinical meetings and consultation } \\
\text { regarding issues related to patient care }\end{array}$ & $1.7 \pm 0.9$ & $31-60$ & $<30$ \\
\hline $\begin{array}{l}\text { Attending to bioinformatics issues } \\
\text { related to patient care }\end{array}$ & $1.8 \pm 0.9$ & $31-60$ & $<30$ \\
\hline $\begin{array}{l}\text { Analysis of test results and } \\
\text { bioinformatics (NGS, CMA) data }\end{array}$ & $2.3 \pm 1$ & $31-60$ & $31-60$ \\
\hline
\end{tabular}

CMA, chromosomal microarray analysis; NGS, next-generation sequencing andex: $1=<30 \mathrm{~min} ; 2=31-60 \mathrm{~min} ; 3=1$ to $2 \mathrm{~h}$; and $4=>2 \mathrm{~h}$. 
with no difference between mean, median, and mode. To clarify, although in this survey our intention was to inquire about patient-related administration, this was not specifically defined in the questionnaire. The modal value for time reported for teaching, research, and other activities was up to $10 \%$ each.

\section{The workload in genetics clinics}

Participants were asked to report the type of patients and how many they see in a typical week. Thirty percent of the geneticists perform only one type of consultation; of these, $74 \%$ are pediatric geneticists (Table 3). Professionals performing one type of consultation have fewer consultation sessions per week $(P<0.05)$. The number of consultations per week among the different professions and among medical geneticists working with and without the support of a genetic counselor was similar; however, geneticists were not asked whether the time they reported and the number of consultations per week included the time and workload of genetic counselors.

\section{The impact of experience on time utilization}

There was no significant difference in the duration of consultation sessions for pediatric consultations, oncogenetic consultations, and consultations regarding fetal malformations between those who had up to 11 years of professional experience and those with more than 11 years of experience, whereas the time reported for sessions related to amniocentesis because of advanced maternal

Table 2 The range of time (in minutes) required for a genetic workup for a pediatric patient, as calculated from the mode of the reports received

\begin{tabular}{lcc} 
& \multicolumn{2}{c}{ Time (min) } \\
\cline { 2 - 3 } Activity & Minimum & Maximum \\
\hline $\begin{array}{l}\text { Literature and database searches related } \\
\text { to genetic consultation }\end{array}$ & 31 & 60 \\
$\begin{array}{l}\text { Clinical meetings and consultations for } \\
\text { issues related to patient care }\end{array}$ & 0 & 30 \\
$\begin{array}{l}\text { Analysis of test results and bioinformatics } \\
\text { (NGS, CMA) data }\end{array}$ & 31 & 60 \\
$\begin{array}{l}\text { Pediatric patient } \\
\quad \text { First genetic consultation session } \\
\quad \text { Summary letter }\end{array}$ & 46 & 60 \\
Total & 1 & 30 \\
\hline
\end{tabular}

CMA, chromosomal microarray analysis; NGS, next-generation sequencing. age or abnormal biochemical screening were shorter for participants with more than 11 years of experience $(P=0.001)$. The time required to write summary letters for all types of consultations was significantly longer for professionals with less than 11 years of experience (pediatric summary, $P=0.007$; oncogenetic summary, $P=0.005$; prenatal summary, $P=0.013$; and amniocentesis summary, $P=0.006$ ). The time required for all other activities was similar for professionals with differing seniority.

\section{Variations between the different professionals practicing medical genetics}

Comparing the responses given by medical geneticists and genetic counselors, there were no significant differences in any category except for the time spent on administration, which was higher for genetic counselors $(P<0.01)$. Among 128 medical geneticists who participated in our survey, 49 stated that they work with the support of a genetic counselor. Comparing the responses given by medical geneticists who work alone with those of medical geneticists who work with genetic counselor support, there were no significant differences in the times reported for the different activities.

\section{The practice of genetics in different continents}

Most comparisons of the amount of time required for genetic consultation sessions and summary letters were similar among the Middle East, Europe, and North America. However, participants from the Middle East reported a longer time required for summary letters for pregnancies with a fetal malformation (when compared with time required by participants from all other regions; $P=0.041$ ) and for summary letters for pediatric patients and for pregnancies with a malformed fetus (when compared with respondents from Europe; $P=0.016$ and $P=0.001$, respectively). They also reported shorter times required for oncogenetic and preamniocentesis consultations compared with North American participants ( $P=0.035$ and $P=0.027$, respectively).

Few significant differences regarding the time required for additional, indirect patient-related activities were observed between the different continents. Middle Eastern professionals reported a longer time required to analyze test results and bioinformatics (NGS, CMA) data, and to search the literature and databases for pathological CMA results compared with all others ( $P=0.03$ and $P=0.01$, respectively), and longer time

Table 3 Number of consultations per week

Consultations $(n)$, by type of professional

\begin{tabular}{|c|c|c|c|c|}
\hline & \\
\hline & All professionals & $\begin{array}{l}\text { Professionals who provide } \\
\text { one type of consultation }\end{array}$ & $\begin{array}{l}\text { Professionals who provide more } \\
\text { than one type of consultation }\end{array}$ & $P$ value \\
\hline \multicolumn{5}{|c|}{ First visit consultations } \\
\hline Minimum & 5.62 & 4.84 & 6 & 0.075 \\
\hline \multicolumn{5}{|c|}{ Total consultations } \\
\hline Minimum & 9.75 & 7.79 & 10.61 & 0.026 \\
\hline
\end{tabular}

Bold values: These were statistically significant $(P<0.05)$. 
for administration compared with the respondents from North America $(P<0.01)$.

Professionals from the Middle East reported more consultations per week. A significant difference in the minimum number of first genetic consultation sessions and minimum total number of consultations was calculated compared with European and North American professionals $(P=0.036$ and $P=0.01$, respectively).

\section{Participants' comments}

The survey respondents added multiple narrative comments reflecting the complexity and challenges they currently face. A representative sample of these remarks, grouped according to theme, is presented here. The profession of each commenter is noted in brackets.

Several respondents pointed out that the survey did not take into account additional clinical activities, such as adult genetics consultations, inpatient consultations, review of charts of other team members, and travel time to outreach locations.

- "This is all outpatients. Inpatient consults-typically intensive care unit-average about $90 \mathrm{~min} /$ patient, including chart review/H\&P [history and physical examination]/counseling. Drive time to outlying facilities." (Medical geneticist (MG))

- "The survey did not include telephone calls to patients for test results, answering questions, etc. between visits and documenting those interactions." (MG)

Many expressed frustration over the workload, the complexity of the profession, and lack of time, including time for research and inadequate compensation.

- "Often overwhelming amount of time that is required to see a patient. Many patients are unique and have complex problems that require large amounts of time. Compensation does not match the time involved." (Medical geneticist working with genetic counselor support (MGWGC))

- "Very complex patients can take several hours of literature or review time. No one else in the medical care system really spends this time." (MGWGC)

- "I think I probably spend as much time outside the clinic as I do with the patient; sometimes it is even more considering the paperwork and "meaningful use" requirements we now have." (MG)

- "Overall, I need about 1.5X as much time outside of clinic for each patient as I spent in clinic." (MGWGC)

- "It is taking longer to see and write reports for patients due to the complexity of the testing available." (MG)

- "This discipline is so wide and demanding." (MG)

- "Time for proper activity is not adequately accounted for. There are increasing pressures to see more patients with less time per patient, yet there are additional time commitments needed on top of a consultation to adequately prepare and counsel a family. This time is not measured per patient but is open ended and is supposed to be within our "admin time." (MGWGC)

- "Yes, it takes a long time to counsel and review the results for a single patient." (MG)

- "In our department it's all about numbers: How many patients you have seen, how quickly you have written your report, etc. Whether you have made the correct diagnosis seems to be less relevant." (MG)

- "Increasing amount of time on administrative tasks and database searching along with the heavy literature researching/reading workload that I previously had, which in some ways is easier because I can access nearly all journal articles online, but there is an ever increasing number to read." (MGWGC)

- "Funding issues have meant cuts to the number of consultant (and GC sessions) resulting in pressure to see larger numbers of cases in a shorter time scale. INSUFFICIENT TIMES ALLOCATED TO GIVE A QUALITY SERVICE TO PEDIATRICS OR COMPLEX CASES!!!” (MGWGC)

- "Spending increasing amount of time researching meaning of array or exome results and also in locating labs to do specific tests at best cost. Lot of paperwork involved in organizing send away genetic tests." (MGWGC)

- "It is hard to set aside time for thoughtful communication to try and do research, but when I can, I really try." (MGWGC)

- "I don't know where I find the time anymore... Something has to give, but I don't know where." (MG)

- "Working at a very busy clinic, one has to devote at least 4 nights till late hours at home on PC to catch up the patient files and summary letters." (MGWGC)

- "I feel I often spend more time on paperwork than actual care of my patients." (Resident)

- "Increasing time required for increasingly complex patients. The genetic counselors see the patients with known diagnosis that I would have seen 10 years ago." (MGWGC)

- "In other words, it is nearly impossible to keep up." (MG)

- Not everyone agreed that there was inadequate allotment of time and compensation. One respondent said, "I think we are hugely lucky to be in a specialty that allows so much time for each patient" (MG).

Others commented on the importance of the survey:

- “Thanks for doing this-It's really important!" (MG)

- "Please publish data, as it is very hard to prove (to administrators) that we need so much time." (MG)

\section{DISCUSSION}

This study, which reflects the experience of genetics professionals around the world, highlights the complexity and challenging aspects of the practice of medical genetics in this era. As hypothesized, the practice of genetics is time consuming, requiring, for 


\section{ORIGINAL RESEARCH ARTICLE}

example, between $1 \mathrm{~h}, 48 \mathrm{~min}$ and $4 \mathrm{~h}$ to work up a pediatric case, including both face-to-face and indirect patient-related activities. Indeed, given the use of new technologies, which require additional time for analyzing test results and bioinformatics, the majority of time is currently spent on indirect genetic consultation-related activities. These time requirements are consistent among professionals practicing medical genetics in different parts of the world and among professionals with different levels of experience.

For a medical geneticist working a full clinical position $8 \mathrm{~h}, 5$ days a week, there might be at most $34 \mathrm{~h}$ of productive work time (allowing for $3 \mathrm{~h}$ of meetings and conferences a week and $3 \mathrm{~h}$ a week for meals, and so on). With an average of $2 \mathrm{~h}$ and $54 \mathrm{~min}$ needed to see a pediatric patient (extrapolated from the minimum and maximum time modes), no more than 11 patients per week can receive a complete workup. In practice, some of the time is allocated to new patients and some to workup related to patients who were previously assessed in the clinic, which requires different time commitments. This calculation does not take into account activities such as research, inpatient consultations, chart review for colleagues supervised by medical geneticists, teaching, and other educational activities.

Previous studies have shown that genetics services are laborintensive, extremely time-consuming, and not self-supporting. ${ }^{1-7}$ Some of these studies evaluated the time used by geneticists alone; others calculated the time required by the complete genetics team, including social workers, clinic coordinators, and secretaries. Also, several studies evaluated only face-toface activities, whereas others also looked into indirect patientrelated activities. Nevertheless, it is obvious from these studies that each case involves extensive time and the income from clinical practice does not cover the expenses. This was calculated as $37 \%$ coverage, according to Bernhardt et al., ${ }^{2}$ or $69 \%$ coverage, according to Bernhardt and Pyeritz. ${ }^{3}$ Given the massive amount of laboratory tests currently available and the massive use of computerized information, most of the time required per case is for indirect activities. According to our survey, only $25-42 \%$ of a clinician's time is dedicated to face-to-face sessions. While the older studies did not stratify the time for direct and indirect activities, it is obvious that in this new era most indirect activity is dedicated to analyses of novel modalities that did not exist previously.

It is not surprising, therefore, that the average genetics professional has about 17 patient encounters per week (both initial and follow-up visits). Given the cost of manpower in medicine, it is unlikely that the direct reimbursement of genetics services can cover such high costs. Some genetic centers have clinical laboratories that generate additional income related to clinical genetic consultation sessions. This should be included in cost-effectiveness calculations. However, many centers do not provide extensive molecular testing services, and with the availability of more advanced and expensive technologies, it may be that fewer, but larger, labs will provide molecular diagnostics for referred patients. In such cases, fewer and fewer genetics services will be supported if based only on financial criteria.
However, the determination of whether the practice of genetics is financially justifiable should not be based only on calculations of the income related to genetics services. Rather, the entire picture should be considered, including the benefits of providing a family with a diagnosis and the appropriate treatments, avoiding unnecessary tests, and assisting families to make informed medical and reproductive decisions. The cost-effectiveness of a molecular diagnosis has been addressed before, as in the case of hereditary hemorrhagic telangiectasia, in which the economic advantages of genetic testing over repeated clinical screening were shown to be substantial. ${ }^{8}$ Similarly, according to the Centers for Disease Control and Prevention, ${ }^{9}$ the total cost of raising a child with medical problems that may be avoided after proper genetic consultation and prenatal or preimplantation genetic diagnosis may be as high as $\$ 51.2$ billion for individuals born in 2000 with mental retardation, $\$ 11.5$ billion for those with cerebral palsy, $\$ 2.1$ billion for those with hearing loss, and \$2.5 billion for those with vision impairment. Given the increasing influence of genetics on the entire field of medicine, the impact of a high-level genetics service on a health-care institution in many domains, such as the quality of care, research, and academic performance, are substantial and should be considered.

The current situation in which genetics services cannot "stand alone" and requires more and more professional tasks and challenges while not being profitable is frustrating. To see more patients and to perform additional academic activities such as research and teaching, medical geneticists often choose to work overtime, and many continue to work evenings and weekends at home. ${ }^{10}$

This situation may account for some of the frustration regarding the working conditions of professionals practicing medical genetics in this era, as was raised by the comments received in this study. The gap between the complexity and effort required for those working in medical genetics and the lack of proper appraisal of individuals and institutes providing genetic services is bound to have a negative effect on the attractiveness of this profession to those considering entering the field. Clearly, the conceptualization of genetic services must change to allow professionals in this field to continue to do this important and challenging work without frustration or an inability to meet the demands necessary to take proper care of patients and families affected with genetic diseases. There is clearly a need for future studies of creative or innovative practice models that improve efficiency or ways that clinical genetic practices justify their work and staffing needs.

To our knowledge, this study is the first attempt to perform an international survey of the time required for various aspects of genetic consultation. It is important to emphasize that this is only a preliminary survey, with a number of limitations. The relatively small number of genetic counselors who answered our survey did not enable us to meaningfully analyze the differences in time allocation of genetic counselors and medical geneticists. We did find that genetic counselors reported significantly more time spent on administrative work, but according to this survey most of the other activities required similar time allocation. Interestingly, when comparing the answers given by medical geneticists who work alone with those of medical geneticists 
who work with genetic counselor support, there were no significant differences in the times reported for the different activities. This is surprising since in certain scenarios and in different types of consultations the genetic counselor and the medical geneticist have different roles and occasionally perform activities that complement one another. Regarding the time requirements for the different activities, we did not ask specifically whether the time reported reflects only the time spent by the physician or also includes time spent by other staff, especially genetic counselors. It could be that the time reported by the medical geneticists in this survey is less than the actual time needed for the entire process of genetic consultation if the portion of counseling conducted by genetic counselors was not included in the reported time.

Other limitations of our study are related to its design, which enabled looking into only a few types of counseling scenarios without analyzing the details of the actual content that needs to be addressed in specific situations. In the current era, the proliferation of choices available to patients can result in a marked increase in genetic consultation durations. For example, in the case of counseling before amniocentesis for advanced maternal age or abnormal screening results, it is appropriate to discuss further issues related to the progress of the field in the 21st century, such as noninvasive prenatal screening options, CMA, methods of rapid numerical chromosomal analysis, and the option to preserve fetal DNA, in order for a couple to make a knowledgeable decision. Also, genetic screening for common severe disorders, such as cystic fibrosis, should be discussed in relevant cases. The level of detail in which genetic counseling regarding familial medical issues is performed is also variable and reflects different policies in different institutes and countries. Similarly, gene panel and genomic tests, which are offered in oncogenetics as well as in other fields, necessitate complex explanations regarding issues such as depth of coverage, inclusiveness, clinical utility when testing genes of varying penetrance and for which there may be no evidence-based management recommendations, and the probability of finding variants of uncertain significance. In different populations, and in different health systems, the ability to offer such testing is variable, and genetic consultation times are probably influenced by this issue. Not only must these novel technological issues be presented and discussed, the different cultural aspects of communication with the patient can be an additional issue that is time consuming as well. These certainly should be addressed in a future survey. The ever-increasing administrative issuesfor example, those related to authorization and coverage for genetic testing, which seem to be a major part of the workload in medical genetics - must be addressed in detail.

We believe that it is important also to transmit the numbers and thoughts presented here to administrators throughout the world to change the perception of genetics services and to establish a background for proper reimbursement. We suggest that the reimbursement process take into account the time needed to evaluate the results of new genomics tests such as CMA and NGS. These tests are extremely powerful and are based on complex, advanced laboratory technologies and bioinformatics tools, but they still require a clinician's knowledge and skills to interpret the results and to determine their clinical implications. Given the obstacles inherent in modern clinical genetics practice, further consideration and assessment are required to determine how to adapt genetic services to meet the demands of cost-effectiveness without compromising the quality of patient care.

\section{SUPPLEMENTARY MATERIAL}

Supplementary material is linked to the online version of the paper at http://www.nature.com/gim

\section{ACKNOWLEDGMENTS}

The authors thank the professionals who participated in this study and the distributors of the mailing lists of the David W. Smith Workshop on Malformations and Morphogenesis, the International Manchester Dysmorphology Conference, and the Israeli Society of Medical Geneticists. The authors also thank Faye Schreiber for English editing.

\section{DISCLOSURE}

The authors declare no conflict of interest.

\section{REFERENCES}

1. Pyeritz RE, Tumpson JE, Bernhardt BA. The economics of clinical genetics services. I. Preview. Am J Hum Genet 1987;41:549-558.

2. Bernhardt BA, Weiner J, Foster EC, Tumpson JE, Pyeritz RE. The economics of clinical genetics services. II. A time analysis of a medical genetics clinic. Am J Hum Genet 1987:41:559-565.

3. Bernhardt BA, Pyeritz RE. The economics of clinical genetics services. III. Cognitive genetics services are not self-supporting. Am J Hum Genet 1989;44:288-293

4. Bernhardt BA, Tumpson JE, Pyeritz RE. The economics of clinical genetics services. IV. Financial impact of outpatient genetic services on an academic institution. Am J Hum Genet 1992;50:84-91.

5. Pletcher BA, Jewett EA, Cull WL, et al. The practice of clinical genetics: a survey of practitioners. Genet Med 2002;4:142-149.

6. Cooksey JA, Forte G, Benkendorf J, Blitzer MG. The state of the medical geneticist workforce: findings of the 2003 survey of American Board of Medical Genetics certified geneticists. Genet Med 2005;7:439-443.

7. McPherson E, Zaleski C, Benishek K, et al. Clinical genetics provider real-time workflow study. Genet Med 2008;10:699-706.

8. Cooksey JA, Forte G, Flanagan PA, Benkendorf J, Blitzer MG. The medical genetics workforce: an analysis of clinical geneticist subgroups. Genet Med 2006:8:603-614.

9. Centers for Disease Control and Prevention (CDC). Economic costs associated with mental retardation, cerebral palsy, hearing loss, and vision impairmentUnited States, 2003. Morb Mortal Wkly Rep 2004;53:57-59. http://www.cdc. gov/mmwr/preview/mmwrhtml/mm5303a4.htm. Accessed February 2015.

10. Bernhardt BA, Zayac C, Trerotola SO, Asch DA, Pyeritz RE. Cost savings through molecular diagnosis for hereditary hemorrhagic telangiectasia. Genet Med 2012;14:604-610. 